\title{
Practical recursive algorithms and flexible open-source applications for planning of smart distribution networks with Demand Response
}

DOI:

10.1016/j.segan.2016.06.004

\section{Document Version}

Accepted author manuscript

Link to publication record in Manchester Research Explorer

Citation for published version (APA):

Martinez-Cesena, E., \& Mancarella, P. (2016). Practical recursive algorithms and flexible open-source applications for planning of smart distribution networks with Demand Response. Sustainable Energy, Grids and Networks, 7, 104-116. https://doi.org/10.1016/j.segan.2016.06.004

Published in:

Sustainable Energy, Grids and Networks

\section{Citing this paper}

Please note that where the full-text provided on Manchester Research Explorer is the Author Accepted Manuscript or Proof version this may differ from the final Published version. If citing, it is advised that you check and use the publisher's definitive version.

\section{General rights}

Copyright and moral rights for the publications made accessible in the Research Explorer are retained by the authors and/or other copyright owners and it is a condition of accessing publications that users recognise and abide by the legal requirements associated with these rights.

\section{Takedown policy}

If you believe that this document breaches copyright please refer to the University of Manchester's Takedown Procedures [http://man.ac.uk/04Y6Bo] or contact uml.scholarlycommunications@manchester.ac.uk providing relevant details, so we can investigate your claim.

\section{OPEN ACCESS}




\title{
Practical Recursive Algorithms and Flexible Open-Source Applications for Planning of Smart Distribution Networks with Demand Response
}

\author{
E. A. Martínez Ceseña* and P. Mancarella \\ School of Electrical \& Electronic Engineering, The University of Manchester, Manchester M13 9PL, UK \\ Eduardo.MartinezCesena@Manchester.ac.uk, +44 (0) 1613068721
}

\begin{abstract}
Distribution networks are currently undergoing fundamental changes due to the rise of smart solutions such as for instance Demand Response (DR), which increases network complexity and challenges the adequacy of traditional planning practices. This calls for the use of suitable planning methodologies. However, the planning problem may be too cumbersome for most commercial software tools, or may lead to complex bespoke optimisation models that may not be easy to use by network planners. In this light, this work proposes a recursive function that can be used in practical algorithms for planning of smart distribution networks. The recursive function can emulate business-as-usual planning practices and further optimise them, including DR options as potential substitutes for network reinforcement. Several case studies based on real UK networks highlight the robustness and flexibility of the proposed algorithms to address different problems, including uncertainty analysis and risk management. The results clearly show that the proposed tool leads to increased economic and social benefits, particularly when optimising investment strategies considering smart DR solutions. The model is herein distributed open-source as accompanying material to this paper with the aim of encouraging new practices required for smart distribution network planning beyond the traditional academic applications.
\end{abstract}

Index Terms - Cost benefit analysis, Demand response, Distribution network planning, Network investment deferral, Recursive functions, Smart grid.

\section{INTRODUCTION}

$\mathrm{S}_{\mathrm{i}}$ IGNIFICANT focus has recently been placed on the facilitate meeting environmental and security of supply targets [1][2]. Distribution networks are thus expected to accommodate different low carbon technologies and increased demand (e.g., due to the electrification of the heating sector [3]), while maintaining high reliability levels needed to foster economic growth. This would normally require massive capital expenditure to upgrade the traditionally passive and fitand-forget based distribution networks. However, new smart active grid solutions such as for instance based on Demand Response (DR) (e.g., through contract-based direct load control activated by aggregators [4] or price-responsive automated DR technologies [5]) could lower the level of investment required [6]. Under the smart grid paradigm, distribution networks will in fact evolve from passive and robust (but expensive) networks to active flexible networks capable of accommodating different resources at lower costs. Nonetheless, this represents a grand challenge for distribution network planning practices, as most traditional planning tools were not designed to handle the levels of flexibility and complexity introduced by the rise of smart active solutions.

In light of the above, recent literature has placed significant focus on new planning tools that can properly model and address the use of DR, automation and communications technologies, and network reconfiguration, amongst other smart solutions [7][8]. The proposed tools rely on significant simplifications and approximations to allow the use of commercial optimisation software (e.g., linear approximations, typical seasonal profiles, limited number of decision nodes, and so forth) [9-11], or in a wide variety of complex algorithms based on meta-heuristic approaches [12-14]. This is still an emerging area of research, as an approach that can capture all the complexity inherent while optimally (and flexibly) planning the distribution networks under real conditions is yet to emerge. In addition, while these tools are indeed bridging the gap between current planning practices and the frameworks needed to adopt smart solutions, their underlying assumption and complex methods make their use in real conditions often difficult. In fact, a general consensus regarding the best tools available to enhance current distribution planning practices has not been reached yet. In any case, Distribution Network Operators (DNOs) are still hesitant to use commercial optimisation software or complex tools, which may favour the use of simple bespoke heuristic approaches.

This work, as part of the UK DNO led Capacity to Customers $\left(\mathrm{C}_{2} \mathrm{C}\right)$ project [15-17], aims at developing a powerful and flexible (but straightforward) distribution network planning methodology based on recursion theory [18][19]. The methodology is meant to facilitate improvements in existing practices and also accommodate emerging smart active solutions (and DR in particular). More specifically, a recursive function that can operate based on two algorithms has been proposed. The first algorithm allows the recursive function to emulate and improve (by systematically assessing available options) current business-as-usual planning practices adopted by DNOs. The second algorithm allows the 
recursive function to operate as an optimisation engine that is robust enough to incorporate complex smart solutions models, and simple enough to be deployed in basic programming languages without the need of dedicated optimisation software. In fact, as recursive algorithms are based on simulations rather than on traditional mathematical programming techniques, DNOs can implement detailed and complex models of different smart grid solutions in the recursive function without concerning for optimisation difficulties that may arise due to the introduction of nonconvex search spaces, integer variables, nonlinear functions, and so forth [20]. In addition, the methodology (particularly of the optimisation engine) is extremely flexible to handle complex planning problems, as will be demonstrated in Section 5.5 by explicitly modelling demand growth uncertainty (i.e., using scenario trees) and risk management (i.e., using the minimax regret approach).

Based on the above, the main contributions of this work are as follows:

1. A simple, flexible and powerful methodology for emulating and even optimising network reinforcement planning practices under realistic conditions is proposed (and provided as open source files to facilitate widespread academic and, particularly, industrial applications).

2. The tool can properly capture the complexity and quantify the value associated with smart solutions emerging at the distribution level such as those based on DR (e.g., the $\mathrm{C}_{2} \mathrm{C}$ method modelled in this work using technical and economic data from real trials) under real conditions and subject to the regulatory framework in place.

3. The flexibility of the tool allows a straightforward implementation of risk management approaches (e.g., stochastic and robust optimisation).

The proposed recursive algorithms have been implemented in an open source tool coded in Matlab [21], which is available as accompanying material of this paper at [22]. Apart from the recursive function (which comprises both recursive algorithms), the accompanying material in [22] includes an input file and a case study. The input file contains information relevant to a real UK distribution network (i.e., the Whalley Range network) such as connectivity, power flows and reliability studies, among other relevant inputs described in Section 4. The case study file illustrates the procedure to initialize and operate the recursive function with the aim of replicating relevant studies presented in this paper (see Section 5).

The rest of the paper is structured as follows. In Section 2, an overview of current distribution network operation and planning practices in the UK is provided. In Section 3, the smart solutions with DR considered within the $\mathrm{C}_{2} \mathrm{C}$ project are discussed in detail. In Section 4 the proposed function comprising two recursive algorithms is presented. Several case studies based on real distribution networks and a wide range of conditions, scenarios and planning problems and approaches are presented in Section 5, while the concluding remarks are given in Section 6.

\section{TRADITIONAL DISTRIBUTION NETWORK PLANNING}

Under current UK standards, the distribution network is planned and operated to meet particular technical and performance requirements (e.g., P2/6 engineering standards [23] and regulated network performance [24]). These requirements are currently assessed with a spreadsheet based Cost Benefit Analysis (CBA) introduced by the UK regulator (the office of gas and electricity markets - Ofgem).

This section provides an overview of current distribution network operation and planning practices, as well as a detailed description of Ofgem's CBA.

\subsection{Current network operation}

Traditionally, high voltage distribution networks in the UK (e.g., $6.6 \mathrm{kV}$ and $11 \mathrm{kV}$ ) are planned using a fit-and-forget approach and predictive security criteria (currently dictated by P2/6 engineering standards [23]). In other words, under current practices, it is assumed that distribution networks are passive and that sufficient redundancy must be in place to guarantee the reconnection of most customers within a reasonable amount of time after a contingency occurs.

A high level description of the operation of the distribution network based on current practices is presented in Figure 1. During normal operation conditions (Figure 1a), the distribution network comprises groups of two or more radial feeders that are interconnected through Normally Open Points (NOPs). If a contingency occurs in one of the feeders, all

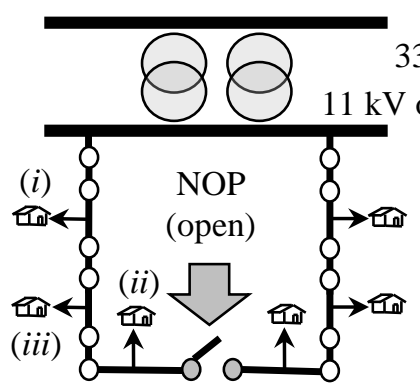

a) Normal operation

(0 minutes)

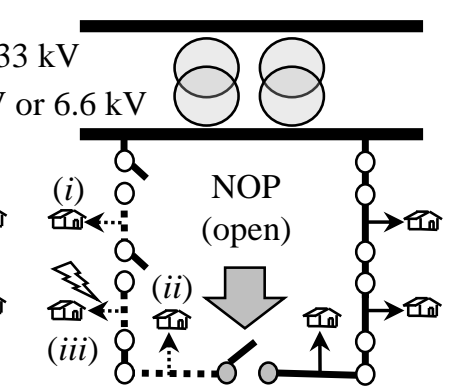

b) Contingency

( $\sim$ minutes)

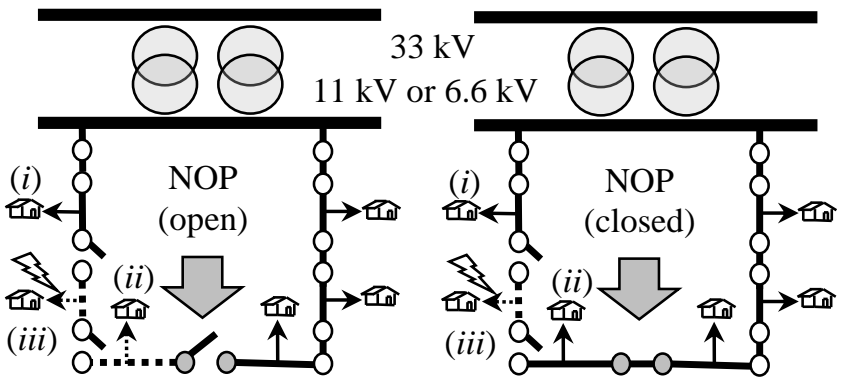

c) Automatic response

( $<3$ minutes)

d) Manual response

( 60 minutes)

Figure 1: Traditional network operations subject to a) normal conditions, b) contingency, c) automatic reconfiguration after a contingency occurs and d) manual reconfiguration while the contingency is cleared. 
customers in that feeder (zones $i, i i$ and iii) would be initially disconnected by the protection system, as shown in Figure $1 \mathrm{~b}$. Afterwards, the protection system isolates the fault while an automated reclosing scheme reconnects some customers that are still connected to their original feeder and isolated form the fault (see zone $i$ in Figure 1c). The automatic response is set to reconnect as many customers as possible within 3 minutes to avoid potential economic penalties, as distribution network reliability is regulated in terms of Customer Interruptions (CI) and Customer Minutes Lost (CML) that last longer than 3 minutes [24]. Then, a repair crew is sent to manually close the NOP to reconnect customers that have become isolated from their original feeder (see zone $i i$ in Figure 1d). Finally, the repair crew would be sent to clear the fault. The customers isolated along the fault (see zone iii in Figure 1d) would be manually disconnected from the fault and either connected to the rest of the network or to a mobile generator if it would take several hours to clear the fault.

This operation and restoration scheme requires additional emergency capacity for each feeder, as a single feeder may be required to supply most (or all) customers in both radials during a contingency. As a result, significant investments in spare capacity are required to maintain current distribution network planning and operation practices.

\subsection{Current distribution network assessment}

Base on the latest UK regulations [25], distribution network upgrades should be assessed with Ofgem's CBA framework [26][27]. Based on Ofgem's CBA, investments at the distribution level should be quantified based on the Net Present Cost (NPC) criterion. The NPC is estimated based on capital and social costs associated with distribution level solutions, as denoted by (1) - (8) [26].

$$
\begin{aligned}
& N P C=\sum_{y=1}^{45} \frac{C_{-} \text {social }_{y}+C_{-} \text {capital }_{y}}{(1+d)^{y}} \\
& C_{-} \text {social }_{y}=C_{-} \operatorname{loss}_{y}+C_{-} \mathrm{co}_{y}+C_{-} c i_{y}+C_{-} \mathrm{cml} l_{y} \\
& C_{-} \text {capital }_{y}=C_{-} \text {expensed }_{y}+D_{y}+C_{-} \text {capitalized }_{y} \\
& C_{-} \text {expensed }_{y}=0.15 \times \sum_{n=1} C_{n, y} \\
& C_{-} \text {capitalized }_{y}=0.85 \times \sum_{n=1} C_{n, y} \\
& \operatorname{Dep}_{y}=\sum_{y 1=1}^{\mathrm{y}} \frac{C_{-} \text {capitalized }_{y 1}}{\text { Dep_lifetime }_{\text {life }}} \\
& C_{-} \text {capital }_{y}=C_{-} \text {expensed }_{y}+\text { Dep }_{y}+C C_{y} \\
& C C_{y}=R A V_{y} \times W A C C
\end{aligned}
$$

$$
R A V_{y}=C_{-} \text {capitalized }_{y}-\text { Dep }_{y}-R A V_{y-1}
$$

where $C_{-}$social $_{y}$ and $C_{-}$capital $_{y}$ are respectively the social and capital costs associated with an investment strategy, $C_{-} l o s S_{y}, C_{-} c o 2_{y}, C_{-} c i_{y}$ and $C_{-} c m l_{y}$ are the costs attributed to power losses, carbon emissions, CI and CML, respectively; $C_{-}$expensed $y_{y}$ and $C_{-}$capitalized $y_{y}$ are parts of the investment $\left(C_{n, y}\right)$ that can be recovered immediately and over time, respectively; $D e p_{y}$ is the depreciation of all capitalized investments divided by the depreciation lifetime (Dep_lifetime), $C C_{y}$ is the cost of capital, $R A V_{y}$ is the regulated asset value, WACC is the pre-tax weighted average cost of capital, the subscript $n$ denotes the n-th intervention associated with a network solution, and the subscripts $y$ and $y$ 1 denote time periods (years).

Ofgem's CBA provides consistent means to assess distribution network reinforcement strategies formulated by different DNOs with the aim of facilitating the regulation of investments at the distribution level. However, the CBA does not include specific solutions (e.g., line and substation reinforcements) or provides a tool to formulate investment strategies.

Accordingly, the DNOs have the flexibility to formulate their own solutions (e.g., DR deployment) and investment strategies. Based on this, and encouraged by economic support to research new distribution network solutions (e.g., the innovation stimulus package [28]), some DNOs are developing new smart distribution network solutions [29], such as the $\mathrm{C}_{2} \mathrm{C}$ method discussed in the next section [15]. It is important to note that the $\mathrm{C}_{2} \mathrm{C}$ method is used here as an example of DR and smart solutions, as other types of solutions can also be modelled and optimised with the proposed recursive function.

\section{Post-Contingency DR: THE $\mathrm{C}_{2} \mathrm{C}$ Method}

The $\mathrm{C}_{2} \mathrm{C}$ method was proposed as a means to reduce capital costs at the distribution level by releasing the previously untapped emergency network capacity during normal operations via the deployment of post-contingency DR. In other words, the $\mathrm{C}_{2} \mathrm{C}$ solution allows the DNO to connect new customers rapidly without the need for network reinforcements even if demand exceeds the firm capacity of the network (e.g., exceeding P2/6 recommendations or N-1 limits in place), as additional post-contingency capacity can be provided by DR if needed. Furthermore, the $\mathrm{C}_{2} \mathrm{C}$ method can also bring several social benefits in the form of reduced power losses, carbon emissions, CI and CML by operating the traditionally radial feeders as closed rings, and automating the NOPs as well as strategic points throughout the network (Figure 2).

In a $\mathrm{C}_{2} \mathrm{C}$ system (Figure 2a), the network is configured as a ring during normal operations by closing the now automated NOP, and automated reclosers are added to disconnect DR customers if needed. This requires investments in the relevant automation and communications infrastructure, as well as the implementation of relevant algorithms to enable post- 


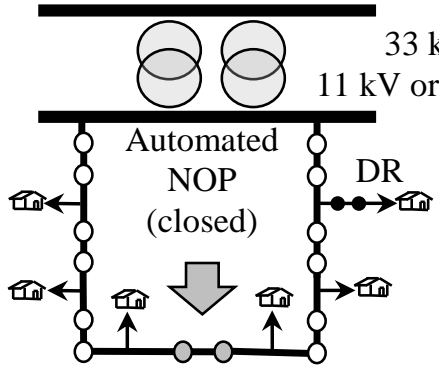

a) Normal operation

(0 mins)

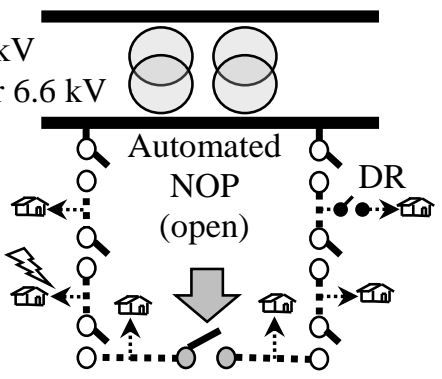

b) Contingency

( $\sim 0$ mins $)$

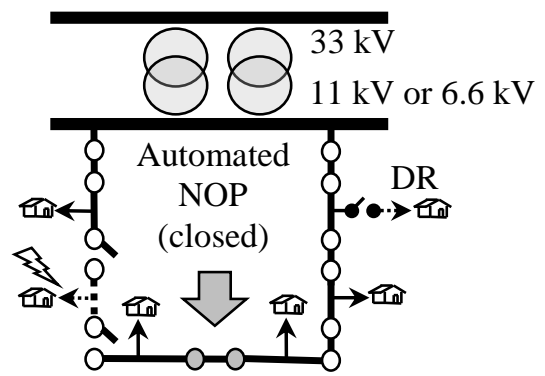

c) Automatic response ( $<3$ mins $)$

Figure 2: Proposed $\mathrm{C}_{2} \mathrm{C}$ configuration subject to a) normal operation conditions, b) the occurrence of a contingency and c) automatic response while the contingency is cleared.

contingency operations.

The ring configuration redistributes the power flows throughout the network, resulting in an immediate reduction in power losses and associated carbon emissions in most cases [30][31]. In addition, the new distribution of power flows can affect the fault level at the point of connection, introduce power flows at the $33 \mathrm{kV}$ level and introduce other technical issues to the networks. Based on existing studies, these issues have been deemed negligible in the 36 representative networks used to trial the $\mathrm{C}_{2} \mathrm{C}$ solution [15][32]. However, these issues might arise and become significant under other conditions, particularly in networks comprising distributed generation. In these cases, suitable power flow and protection studies should be carried out to fully address all the techno-economic implications of applying the presented DR solution.

In this case, if a contingency occurs, all customers in both feeders would be initially disconnected (Figure 2b). This results in an increase in short-term interruptions for less than 3 minutes. However, due to the increased automation levels, most costumers can be restored within 3 minutes (Figure 2c); effectively decreasing the regulated $\mathrm{CI}$ and CML. A description of the restoration protocols based on the enhanced automation and information infrastructure associated with the $\mathrm{C}_{2} \mathrm{C}$ method can be found in [15][33]. Information from Trials to date suggest that the increased short-term interruptions have not been noticed by customers, potentially due to the low frequency of contingencies (once every three years or even less frequently) [34].

In light of this, the $\mathrm{C}_{2} \mathrm{C}$ method is a DR application that allows DNOs to operate the networks beyond traditional security limits by, for instance, allowing (immediate) new connections without investing in costly additional network security capacity. Security limits are still met by disconnecting DR customers during emergency conditions whenever needed. This does not imply that DR customers will be disconnected after every contingency occurs. In fact, DR would only be needed if the contingency occurs near the yearly peak times when demand is close to the security constrained firm capacity of the network. Considering that these peak time conditions only occur a couple of hours per year (typically in winter in the UK) and that contingencies are infrequent (one event every three years or less frequently), DR would seldom be required. Regardless, DR customers would still receive periodic payments for their availability, which can make postcontingency DR attractive for some customers [15][35][36]. It is worth noting that all customers connected to the $\mathrm{C}_{2} \mathrm{C}$ trial networks were considered potential DR providers. That is, $\mathrm{C}_{2} \mathrm{C}$ contracts were offered to existing and new customers subject to the DR needs of the network (i.e., DR is not contracted when it is not required). These customers were offered reduced connection charges (for new customers), lower distribution fees, direct payments and other combinations of incentives with an average value of 23 $\mathrm{k} £ / \mathrm{MW}$ peak per year (see [37] for an overview of customer engagement studies and payment options).

In this work, in line with what is actually implemented in the $\mathrm{C}_{2} \mathrm{C}$ project, the DR costs $\left(C_{D R}\right)$ are assumed to comprise an investment in an enhanced automation and reconfiguration scheme for the network $\left(C_{D R_{\text {Network }}}\right)$, customer automation $\left(C_{D R_{\text {Customer }}}\right)$ and an annual payment for DR customers $\left(C_{D R, y}\right)$, as shown in (9).

$$
C_{D R}=C_{D R_{\text {Network }}}+C_{D R_{\text {Customer }}}+\sum_{n=1} C_{D R, y}
$$

These costs are introduced into Ofgem's CBA as parts of the investments associated with one of the $\mathrm{n}$-th solutions $\left(C_{n, y}\right)$ (i.e., the $\mathrm{C}_{2} \mathrm{C}$ solution in this case).

\section{RECURSION BASED NETWORK PLANNING METHODOLOGY}

The introduction of DR (and other smart active solutions) adds complexity to the formulation of distribution network upgrade strategies, as DR could be deployed as an alternative to or in combination with traditional line and substation reinforcements. The latter may be particularly attractive as DR can defer or avoid costly upgrades, whereas low cost upgrades could reduce the amount of DR eventually required.

Two algorithms deployed in a bespoke recursive function are proposed in this work as a means to emulate business-asusual planning practices and optimise (using a novel optimisation engine) investment strategies in smart distribution networks. The recursive function can be implemented in most commercial programming environments (e.g., C++, Java) and has been specifically implemented in an open source Matlab tool. The tool, which is available at [22] as accompanying material to this paper, can thus enable DNOs to perform comprehensive business-as-usual planning 
practices automatically, as well as complex optimisations without the need of dedicated optimisation software. Furthermore, since, as aforementioned, recursive functions are based on simulations rather than formal mathematical programming techniques, DNOs can readily implement detailed and relatively complex models of different solutions (e.g., the $\mathrm{C}_{2} \mathrm{C}$ method) and regulatory frameworks (e.g., Ofgem's CBA).

This section provides a general overview of the proposed recursive function, and its permutations for smart distribution network planning (considering DR options) based on the "business-as-usual" and "optimisation" algorithms.

\subsection{Overview of the proposed recursive function}

Generally speaking, a recursive function is a function that creates copies of itself (instances) [18][19]. This type of functions can solve a problem by dividing it into smaller subproblems that are addressed by "child" instances. Each "child" instance further divides the problems into even smaller subproblems addressed by additional "child" instances, and so forth. Eventually, termination conditions are reached when the sub-problems are small enough to be solved, and each subproblem has been addressed by an instance. At this point, each instance reports the solution of its sub-problem to its "parent" instance, which devises a solution (for a larger sub-problem) using the information provided by all its "child" instances and reports this solution to its own "parent" instance. This process is repeated until the information reaches the original recursive function, which receives enough information to solve the complete problem. Accordingly, recursive functions can solve large problems using information from the collaborative operation of all instances [18][19]. That is, a relatively simple recursive function can handle large and complex problems, as the problem addressed by each instance is greatly simplified.

It is important to note that iterative approaches can be used as an alternative to recursive functions [38][39]. On the one hand, iterative approaches may be faster and easier to code than recursive functions for some applications. On the other hand, the simplifications made by recursive functions (i.e., each instance only addresses a given set of interventions subject to a specific load profile) can be easier to understand and more suitable to the operation of DNOs. Furthermore, (i) computational complexity is not an issue for the proposed algorithm under the realistic conditions explored in this work (see Section 5.4), as it can normally find solutions within a couple of minutes, and (ii) recursive functions tend to facilitate the analysis of decision trees, which is critical for the potential future applications of the proposed approach explored in Section 5.5.

In light of the above, this work proposes a methodology based on a simple recursive function that can address the complex distribution network planning problem. Generally speaking, the proposed methodology (see Figure 3) consists of preparing all inputs required by the function, and initialising and calling the recursive function (see also the case study file in [22]).

The inputs considered in this work are based on the

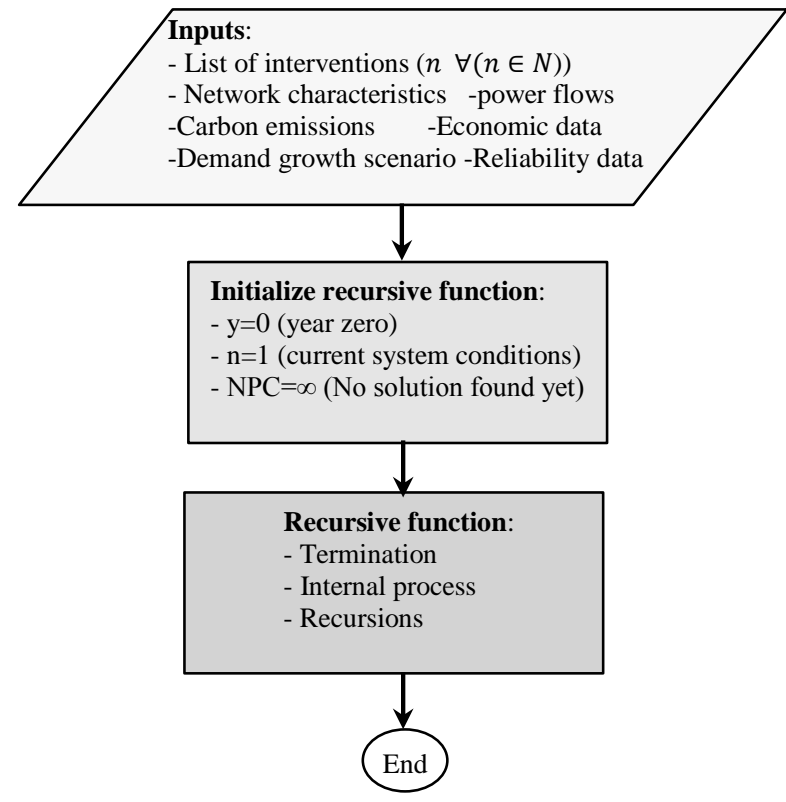

Figure 3: High level flow diagram of the proposed method.

information that would normally be collected by DNOs via a preliminary network analysis to populate Ofgem's CBA framework [40]. More specifically, the inputs are calculated off-line and comprise a list of interventions selected for the study (e.g., network and substation upgrades and the $\mathrm{C}_{2} \mathrm{C}$ method), as well as information relevant for the calculation of power flows (e.g., for the estimation of power losses, and network and substation capacity voltage, thermal and security limits), network reliability, carbon emissions and economic indicators in light of a particular demand growth scenario. This information should and can readily be updated within the accompanying Matlab scripts if other solutions or assessment frameworks are to be used (see also the input file in [22]).

Once the inputs have been defined via off-line studies, the recursive function is initialised by setting the initial year of study to zero $(y=0)$, and defining that none of the n-th potential interventions has been recommended $(n=0)$ and a solution is yet to be found $(N P C=\infty)$. The instance that receives this information defines itself as the original recursive function and creates instances of itself (children, grandchildren and so forth) to find an investment strategy, which comprises a set of recommended interventions throughout every year defined in the load growth scenario. For this purpose, the recursive function and all of its instances use the following three stages process (see Figure 3 and Figure 4):

1. Internal process: At this stage, the recursive function (or any instance) models the distribution network in a single specific year. For example, the original recursive function would assess the current network without interventions under existing demand conditions (i.e., year zero). The assessment involves the estimation of costs, power losses and other parameters relevant to Ofgem's CBA (see (1)-(9)) for the specific network under assessment in a particular year. This process also involves assessing the feasibility of the network in terms of voltage, thermal and security limits. 
2. Recursions: The operation of the recursive function varies depending on whether the function is using the business-as-usual or the optimisation algorithms (this is discussed in detail in the next subsections). However, generally speaking, at this stage the function formulates different combinations of the $\mathrm{n}$-th available interventions denoted in a list $n \forall(n \in N)$ (e.g., doing nothing, deploying the $\mathrm{C}_{2} \mathrm{C}$ method, reinforcing the network and/or upgrading the substation) and creates "child" instances to assess them. Following the example, the original recursive function would create "child" instances to assess each available intervention or combination of interventions in the next year (year 1). Each "child" instance would create additional "child" instances to assess interventions in year 2 and so forth until termination conditions are met.

3. Termination: An instance terminates if (i) it has reached the end of the planning horizon (45 years for UK distribution networks), (ii) it is not allowed to create "child" instances or (iii) all its "child" instances have terminated. Reaching the end of the planning horizon implies that a feasible investment strategy has been formulated; in which case the instance assess the investment strategy (based on Ofgem's CBA in this work; although other approaches can be used) and reports the results to its "parent" instance (e.g., NPC and investment strategy). An instance may not be allowed to create "child" instances based on constraints imposed in the business-as-usual or optimisation algorithms (discussed below); in which case the instance reports that a feasible investment strategy could not be found (i.e., $\mathrm{NPC}=\infty$ ). Finally, an instance may receive feedback from all its child instances (i.e., NPCs and investment strategies); in which case it reports the most attractive result (investment strategy with the lowest NPC) to its "parent" instance.

This three stages process is used for two planning applications, namely, to emulate business-as-usual practices as well as to optimise investment strategies based on two algorithms, as is discussed in detail in the following subsections.

\section{2. "Business-as-usual” recursive algorithm}

Based on business-as-usual practices, the distribution networks are only upgraded whenever the normal operation of the network becomes infeasible; moreover, only the cheapest available solutions are implemented. That is, capital investments are only made to upgrade networks whenever additional firm capacity (or in case DR as an example of smart active solution) is needed to ensure that the networks meet technical and performance standards (as dictated by regulations and P2/6 engineering recommendations in the UK). The recursive algorithm proposed to emulate businessas-usual practices uses the logic presented in Figure 4 when the switches are turned to the right (i.e., connecting the lines marked as "Business-as-usual mode").

In this case (see Figure 4), the recursive function (and all its

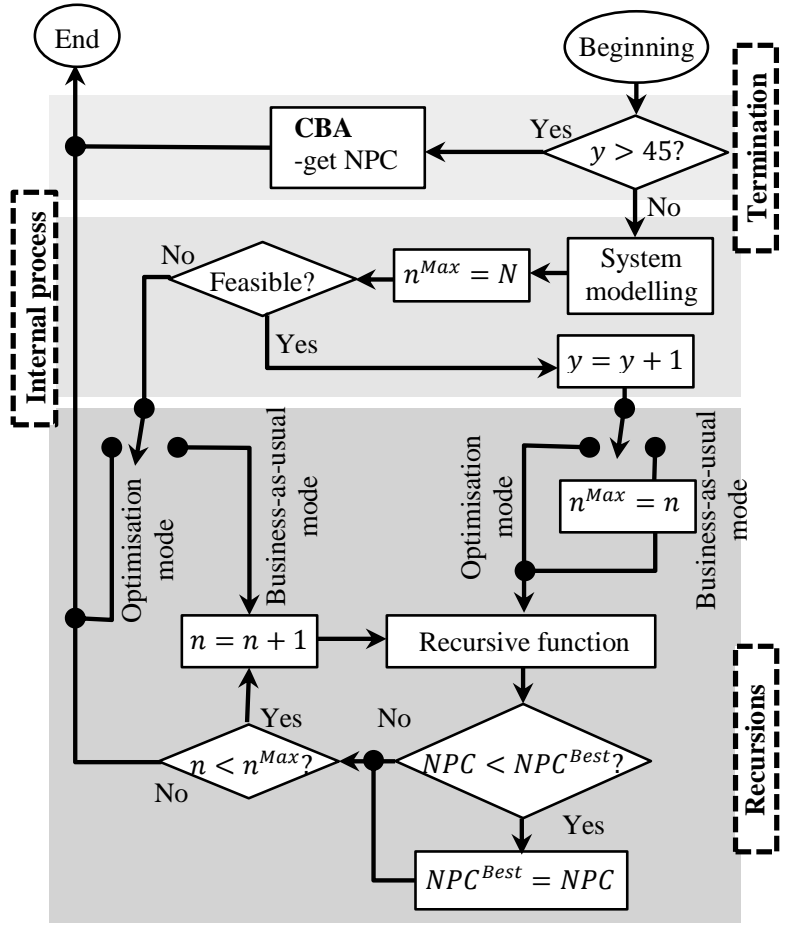

Figure 4: High level flow diagram of the recursive function used under either business-as-usual or optimisation mode.

instances) would only propose interventions when needed to meet thermal, voltage or security considerations (i.e., feasibility). For example, assume that the current network is feasible throughout the demand growth scenario considered. In this case, after initialisation (see Figure 3), the function would begin by modelling the network in year zero. As the network is feasible in this year, the function would only create one instance to assess the current network in year one (this is achieved by setting $n^{\operatorname{Max}}=n$ ). All subsequent instances would do the same until reaching termination conditions (in year 45 , in the specific case); in which situation they would report that the best alternative (the only alternative considered in this case) is to avoid deploying any intervention. Now assume that the network is infeasible in a given year. In such a case, the relevant instance would consider the deployment of the n-th available interventions $\left(n^{\operatorname{Max}}=N\right.$ or a subset of $N$ ) as a means to meet feasibility, and would call "child" instances to address each potential intervention. This time, several instances may reach termination conditions; in which case several investment strategies with different associated NPCs would be identified. The relevant "parent" instances (ultimately the original recursive function) would select the investment strategy with the lowest NPC.

This operation is in line with business-as-usual practices as interventions are only triggered by feasibility considerations and only the lowest cost investment strategy is selected. However, the proposed approach can be considered an improvement compared with existing planning approaches, as it systematically assesses a wide range of available alternatives. In practice, only a limited number of investment strategies may be considered, as the project planner may have to formulate and assess each strategy manually. 


\section{3. "Optimisation" recursive algorithm}

The proposed recursive function can operate as an optimisation engine using the logic presented in Figure 4 when the switches are turned to the left (i.e., connecting the lines marked as "Optimisation mode").

The optimisation technique used by the recursive function is similar to an exhaustive search as it considers all potential combinations of interventions throughout the planning horizon. On the one hand, this is the most straightforward and robust optimisation technique available as it is simple to understand (and easy to explain to DNOs, regulators and other actors) and guarantees finding the optimal solution under all conditions (even for complex mixed integer, nonlinear and nonconvex problems). On the other hand, this approach tends to be overlooked (particularly for academic applications) due to its simple nature and because it can be computationally expensive or even infeasible in most applications [41][42]. In this light, the recursive function has been designed to significantly reduce the computational costs of the search by systematically terminating all instances that face an infeasible investment strategy (e.g., in this work, whenever the network fails to meet $\mathrm{P} 2 / 6$ recommendations), which also prevents them from spreading. In other words, rather than using a full exhaustive search that can be computationally prohibitive, the recursive function uses a smarter reduced search that minimises computational burden while still guaranteeing optimality. Thanks to this adjustment, the methodology modifies the simple and clear exhaustive searches for modelling the complex distribution planning problem under realistic conditions.

As an example, assume again a network that is feasible throughout the planning horizon. After initialisation (see Figure 3), the original recursive function would create "child" instances to assess the different potential interventions to be deployed in year 1 (i.e., considering $n^{\operatorname{Max}}=N$ ). Most of these "child" instances would have fewer alternatives to upgrade the system as some interventions would become infeasible (e.g., NOP automation cannot be deployed twice, lines cannot be reinforced to a lower capacity level, and so forth). Accordingly, the number of feasible interventions drops rapidly after a few generations of "child" instances. Now consider that the current distribution network is infeasible in some years subject to the demand growth scenario, as would be likely the case if the DNO is assessing alternatives to upgrade the system. This would lead to many infeasible conditions that would result in the early termination of numerous instances (which would not spread) and thus to a reduction of the search space and computational burden as only a few instances would reach termination at the end of the planning horizon. As in the business-as-usual case, after reaching termination conditions, the instances report their findings to their "parent" instances and this information exchange procedure ultimately allows the recursive function (now in optimisation mode) to identify the most attractive investment strategy.

Based on the above, the computational burden associated with the application of the proposed optimisation algorithm is expected to be modest when assessing realistic distribution networks that may require upgrades in the short-term. Further discussion is provided below (i.e., Section 5.4), where the time complexity of the algorithms is tested under different conditions.

\section{CASE STUdiES}

In this section, a real UK distribution networks (i.e., one of the $36 \mathrm{C}_{2} \mathrm{C}$ trial networks) is used to illustrate the capabilities of the proposed methodology to emulate business-as-usual practices and optimise investment strategies also considering DR options. It is shown that the use of an optimisation engine greatly increases the economic attractiveness of smart DR based solutions (e.g., the $\mathrm{C}_{2} \mathrm{C}$ method in this case). Afterwards, the robustness of the approach to handle different types of systems and the associated time complexity are tested by extending the case study to all $36 \mathrm{C}_{2} \mathrm{C}$ trial networks. Finally, the flexibility of the methodology (particularly of the optimisation engine) to handle complex planning problems is shown by explicitly modelling demand growth uncertainty (i.e., using scenario trees) and risk management (i.e., using the opportunity loss criterion also known as minimax regret).

\subsection{Case study description}

As showed in Figure 3, the first step of the proposed methodology is to define the relevant inputs for the assessment of a particular distribution network based on the characteristics of the network and several preliminary analyses.

For this example, consider the Whalley Range network, which is a $6.6 \mathrm{kV}$ distribution network comprising 10 feeders (32 nodes) connected to a $26.50 \mathrm{MW}$ substation. This study focuses on two of these feeders (see Figure 5), which are currently being used for $\mathrm{C}_{2} \mathrm{C}$ trials and supply 4131 customers (mainly urban customers).

The DNO responsible for this network (i.e., ENWL) envisions three potential demand growth scenarios, which may result in demand exceeding the firm capacity of the substation and lines of this system as shown in Figure 6 [43].

It can be seen in the figure that firm capacity of the lines is expected to be reached after demand grows by $3 \%$ and, if the lines are reinforced (i.e., in this case up to $800 \mathrm{~m}$ of the $3 \mathrm{~km}$ of lines - mostly near the point of connection - can be reinforced), firm capacity would be reached once again after a $22 \%$ demand growth. The costs of the first and second line

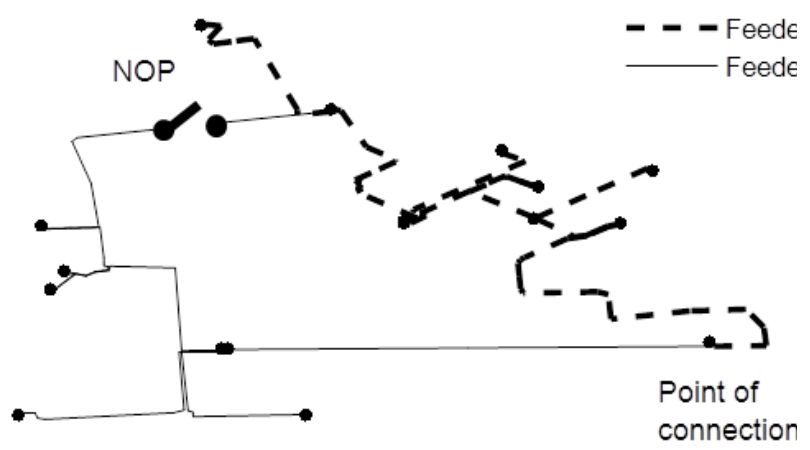

Figure 5: The Whalley $6.6 \mathrm{kV}$ distribution network. 


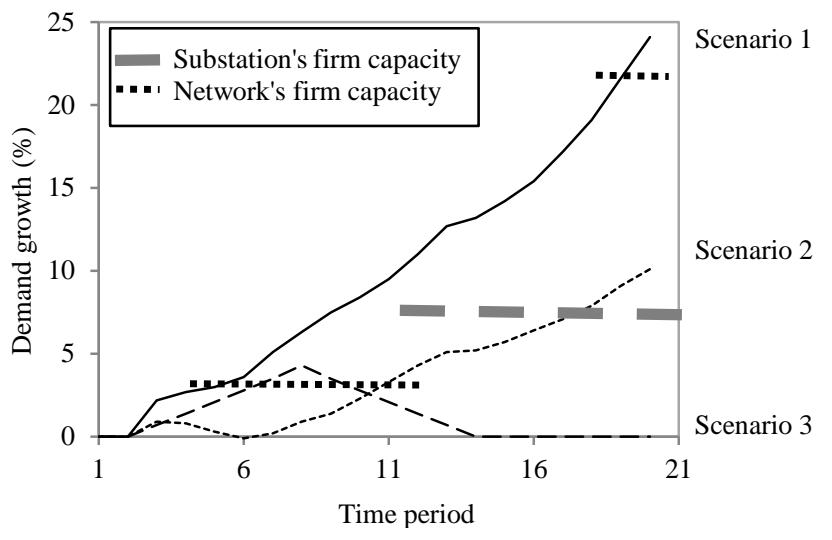

Figure 6: Scenarios considered for the study.

reinforcements are estimated to be $63 \mathrm{k} £$ and $84 \mathrm{k} f$, respectively, and the associated planning and construction lead time is expected to be two years. Substation firm capacity would instead be reached after demand increases by $10 \%$. Reinforcing the substation costs $338 \mathrm{k} £$ and takes roughly three years.

The network and substation costs and capacities were determined based on inputs from ENWL and conventional AC power flow calculations (MatPower [44] was used; however, other tools could be used). For this purpose the hourly power flows of the network were sequentially simulated throughout a year (i.e., 8760 simulations) in light of the demand growth scenarios (three scenarios for 45 years) ${ }^{1}$. Firm capacity was thus determined based on thermal and voltage limits, and security considerations based on P2/6 criteria [23] (i.e., assuming the potential failure of the lines connected to the point of connection) and emergency ratings (i.e., the elements can withstand a $20 \%$ overload above their firm capacity for up to two hours within a 24 hours period). The power flow calculations (only for normal operation) were used to determine relevant power losses associated with the substation and the network. See Figure 7 for an overview of the power losses associate with different configurations and reinforcements.

The characteristics of the considered DR intervention were determined based on information from the $\mathrm{C}_{2} \mathrm{C}$ trial networks to date. Accordingly, the DR intervention costs are assumed to comprise (i) a single payment of $19 \mathrm{kf}$ for upgrading the network's automation levels (including automating the NOP), (ii) a single payment of $24 \mathrm{kf} / \mathrm{MW}$ for customer automation and (iii) an annual payment of $23 \mathrm{kf} / \mathrm{MW}$ for the availability, administration and billing of peak DR capacity. The lead time for planning and deploying the DR intervention is estimated to be roughly a year. It is considered that there is sufficient DR capacity available to meet future demand needs without reinforcing the network in any scenario; although it may be costly to deploy the required amount of DR in some cases.

The CI and CML reliability indices were calculated based on sequential Monte Carlo simulations subject to different levels of DR availability (expressed in \%) and considering

\footnotetext{
${ }^{1}$ For the sake of computational efficiency, overlapping power flows associated with the same demand growth in different years and scenarios were taken from the same simulation.
}
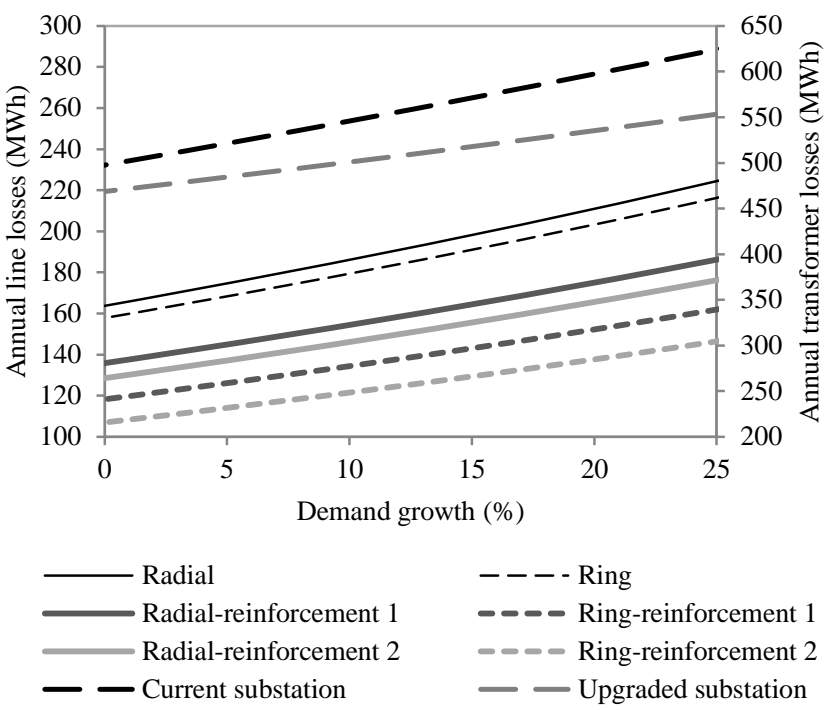

Figure 7: Expected power losses associated with different network configurations, upgrades and reinforcements.

typical radial networks without automation and ring networks with increased automation levels [45]. However, in practice, estimates of these parameters based on DNO's experiences may also be used. An example of the CI and CML calculated for this case study is presented in Figure 8 .

Based on recommendations and inputs from Ofgem [26][27], power losses are priced at $48.42 £ / M W h, C I$ at $£ 15.44$ per interruption and CML at $£ 0.38$ per minute lost. Annual forecasts for greenhouse gasses conversion factors (e.g., $0.503 \mathrm{t} / \mathrm{MWh}$ in 2016) and traded carbon prices ( $(\mathfrak{t} / \mathrm{t}$ in 2016) were also taken from Ofgem's CBA [26]. See [22][40] for further details on the inputs needed to populate Ofgem's CBA and the recursive function used in this study.

\subsection{Results from the recursion algorithms}

After being initialized (see Figure 3), the proposed recursive function first uses the business-as-usual algorithm to formulate Traditional and Active investment strategies. The Traditional investment strategies (Table I) comprise line and

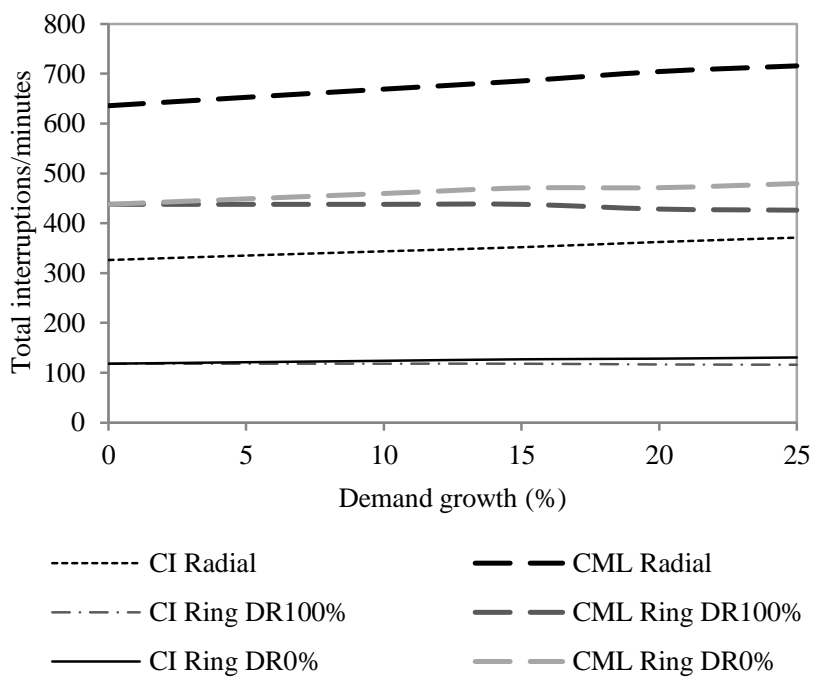

Figure 8: Example of CI and CML associated with different network configurations and automation levels, and use of DR. 
substation reinforcements deployed before reaching firm capacity subject to planning and construction lead times. Similarly, the Active strategies (Table II) avoid traditional interventions by deploying DR interventions. In practice, DNOs would use the business-as-usual approach to assess different strategies to select the most attractive alternative. Accordingly, a Smart strategy for the business-as-usual case is formulated as the best alternatives between the Traditional and Active strategies in terms of their associated NPC.

As a second step, the recursive function uses the optimisation algorithm to improve the Traditional and Smart strategies (considering decision nodes for the first 20 years of the planning horizon). For example, the optimised Traditional (OTrad) strategy now has the flexibility to perform interventions and oversize reinforcements to mitigate social costs even before reaching firm capacity. As shown in Table III, this flexibility allowed the OTrad strategy to oversize the network in Scenario 1.

The optimised Smart (OSmart) strategy has the flexibility to combine traditional and active solutions. As shown in Table IV, this strategy tends to combine the different solutions and even deploy them before firm capacity is reached. This is expected to provide economic gain from deferred (or avoided) reinforcements and reduced DR costs, as well as significant social benefits in terms of losses reductions as shown in Figure 7. For example, when performing DR interventions before firm capacity is reached, only network automation costs are initially incurred, as the associated annual availability payment for DR customers (and customer automation) can be postponed until firm capacity is reached.

The performance of the different investment strategies in the different scenarios in terms of their NPC are presented in Table V.

Based on business-as-usual practices, the Traditional strategy only outperforms the Active strategy in Scenario 1 where significant demand growth results in high DR availability payments. In other scenarios, more modest DR payments allow the deferral and/or avoidance of some traditional solutions, which makes the Active strategy more economically attractive than the Traditional strategy. In this case, DR interventions are only considered as alternatives to potentially costly traditional reinforcements, which results in deploying the Traditional strategy in Scenario 1 and the Active strategy in Scenario 2 and Scenario 3 as a Smart strategy for business-as-usual practices.

Based on the optimised strategies, it can be seen that optimising the Traditional strategy provides little value (only in Scenario 1 in this example). This is attributed to the little value to be gained from the flexibility to deploy costly traditional line and substation reinforcements before firm capacity is reached. However, once DR interventions are also considered, the optimisation engine can formulate economically attractive combinations of traditional and active smart solutions deployed at different times (e.g., deploying DR interventions to avoid costly reinforcements and deploying the cheapest reinforcements to mitigate DR costs). As a result of this flexibility, the OSmart strategy outperforms all other
Table I: Description of the Traditional investment strategy.

\begin{tabular}{|l|l|}
\hline Scenario & Description \\
\hline 1 & $\begin{array}{l}\text { Reinforce the lines in year 4, upgrade the substation in year } 9 \\
\text { and reinforce the lines again in year } 18 .\end{array}$ \\
\hline 2 & Reinforce the lines in year 9 and the substation in year 17. \\
\hline 3 & Reinforce the lines in year 5. \\
\hline
\end{tabular}

Table II: Description of the Active investment strategy.

\begin{tabular}{|l|l|}
\hline Scenario & Description \\
\hline 1 & DR intervention in year 4. \\
\hline 2 & DR intervention in year 9. \\
\hline 3 & DR intervention in year 5. \\
\hline
\end{tabular}

Table III: Description of the OTrad strategy.

\begin{tabular}{|l|l|}
\hline Scenario & Description \\
\hline 1 & $\begin{array}{l}\text { Reinforce the lines in year } 4 \text { (double reinforcement), upgrade } \\
\text { the substation in year 9. }\end{array}$ \\
\hline 2 & Reinforce the lines in year 9 and the substation in year 17. \\
\hline 3 & Reinforce the lines in year 5. \\
\hline
\end{tabular}

Table IV: Description of the OSmart strategy.

\begin{tabular}{|l|l|}
\hline Scenario & Description \\
\hline 1 & $\begin{array}{l}\text { DR intervention in year 1, reinforce the lines in year } 4 \\
\text { (double reinforcement) and upgrade the substation in year } \\
13 .\end{array}$ \\
\hline 2 & DR intervention in year 1 and line reinforcement in year 9. \\
\hline 3 & DR intervention in year 1. \\
\hline
\end{tabular}

Table V: NPC comparison of the different strategies.

\begin{tabular}{|l|l|l|l|l|l|}
\hline \multirow{2}{*}{ Scenario } & \multicolumn{5}{|l|}{ NPC $\left(\left(_{\left.\mathbf{~} ~ \mathbf{~ 1 0}^{3}\right)}\right.\right.$} \\
\cline { 2 - 6 } & Traditional & Active & Smart & OTrad & OSmart \\
\hline 1 & 918 & 1229 & 918 & 899 & 794 \\
\hline 2 & 757 & 718 & 718 & 757 & 527 \\
\hline 3 & 545 & 475 & 475 & 545 & 465 \\
\hline
\end{tabular}

Table VI: Potential NPC savings (\%) from using optimised strategies.

\begin{tabular}{|l|l|l|l|l|l|}
\hline \multirow{2}{*}{ Scenario } & $\begin{array}{l}\text { OTrad savings } \\
\text { compared with: }\end{array}$ & \multicolumn{4}{|l|}{ OSmart savings compared with: } \\
\cline { 2 - 6 } & Traditional & Smart & Traditional & Active & OTrad \\
\hline 1 & $2 \%$ & $13 \%$ & $13 \%$ & $35 \%$ & $12 \%$ \\
\hline 2 & $0 \%$ & $27 \%$ & $30 \%$ & $27 \%$ & $30 \%$ \\
\hline 3 & $0 \%$ & $2 \%$ & $15 \%$ & $2 \%$ & $15 \%$ \\
\hline
\end{tabular}

strategies in all scenarios, as shown in Table V and Table VI. This highlights that the use of an optimisation engine is particularly attractive in light of smart active solutions such as the $\mathrm{C}_{2} \mathrm{C}$ method. It is worth noting that the results presented in this section can be replicated using the case study file provided in [22].

\subsection{Sensitivity studies}

With the aim of testing the robustness of the proposed recursive function, the case study is extended here to all 36 $\mathrm{C}_{2} \mathrm{C}$ trial networks under different conditions. More specifically, sensitivity studies of the key assumptions (i.e., line and DR costs) are performed to demonstrate the robustness of the proposed tool, as well as to highlight the conditions that encourage or discourage the use of the DR and the optimisation algorithm.

The sensitivity studies address normal conditions based on current costs estimates for each network, as well as two adverse conditions for DR. The adverse conditions represent cases where traditional line reinforcement costs are reduced to only $25 \%$ of their estimated value and DR costs are increased 
to $200 \%$ of their original value. These conditions challenge the DR intervention, which is a low cost alternative to defer or avoid costly network interventions. It is important to note that, even though other key parameters, such as substation headroom and DR availability could be addressed (see [43] for additional sensitivity studies using other tools), the most critical conclusions for this work can be drawn from the three conditions selected.

The results in Table VI and Table VII show that, under normal conditions and using the business-as-usual algorithm, the performance of the Traditional and Active strategies and the associated Smart strategy follow the same trend as in the previous case study. However, in other cases, the DR intervention becomes unattractive as an alternative to avoid cheap and few reinforcements (Scenario 3 under 25\% line costs conditions) or too expensive to compete with typically costly reinforcements (Scenario 2 under 200\% DR costs conditions). This implies that, in order to deploy smart solutions under business-as-usual practices, it is critical to identify the conditions that make these solutions economically attractive. For instance, even if a DR intervention is deemed attractive in Scenario 2 under current conditions, DNOs would be hesitant to follow this strategy if DR costs are uncertain as the solution may become too costly in the future.

Now, using the optimisation algorithm to improve the different strategies, it can be corroborated that optimising the Traditional strategy to create the OTrad strategy provides little value under most conditions. Conversely, the OSmart strategy, which considers both traditional and active smart solutions, is still significantly more attractive than other strategies, even subject to adverse conditions for DR interventions (see Table VIII).

The results in Table VIII show that the OSmart strategy outperforms all other solutions by at least $15 \%$ in all cases considered in this study. This strategy can be significantly valuable under both adverse and favourable conditions for DR interventions, as it can combine active smart and traditional solutions to limit the costs of either solution should it become capital intensive. These results clearly show that the use of simple but practical planning methodologies such as the one presented in this work can facilitate significant costs savings for DNOs (and customers) via an improved utilization of smart solutions compared with business-as-usual practices.

\subsection{Computational complexity}

The sensitivity studies presented above (i.e., 324 simulations comprising 36 networks assessed in 3 scenarios under 3 different conditions) were performed in roughly $21 \mathrm{~h}$ using an i5-3470 $3.2 \mathrm{GHz}$ processor and 4GB of RAM. In addition, $10 \mathrm{~h}$ of simulations were required to perform the offline studies required for populating the recursive function (see inputs file in [22]). These off-line studies included (i) loading network and solution data (from excel) and formatting the data to meet Matlab requirements, (ii) formulating profiles with an hourly resolutions for the annual demand growth forecasted in each scenario, (iii) performing power flow studies based on the demand profiles (hourly resolution for every year in the
Table VII: Average expected NPC performance of the different strategies in the 36 trials and networks subject to different conditions.

\begin{tabular}{|l|l|l|l|l|l|l|}
\hline Conditions & \multirow{2}{*}{ Scen. } & \multicolumn{2}{|l|}{ NPC $\left(\mathbf{f x 1 0}^{\mathbf{3}}\right)$} & \multicolumn{4}{l|}{ OSmart } \\
\cline { 2 - 7 } & & Traditional & Active & Smart & OTrad & OS4 \\
\hline \multirow{3}{*}{ Normal } & 1 & 1164 & 1373 & 1164 & 1159 & 945 \\
\cline { 2 - 7 } & 2 & 890 & 811 & 811 & 889 & 588 \\
\cline { 2 - 7 } & 3 & 597 & 555 & 555 & 597 & 469 \\
\hline \multirow{2}{*}{$\begin{array}{l}25 \% \text { line } \\
\text { costs }\end{array}$} & 1 & 1045 & 1373 & 1045 & 1043 & 838 \\
\cline { 2 - 7 } & 2 & 819 & 811 & 811 & 819 & 518 \\
\cline { 2 - 7 } & 3 & 519 & 555 & 519 & 519 & 423 \\
\hline \multirow{2}{*}{$\begin{array}{l}200 \% \text { DR } \\
\text { costs }\end{array}$} & 1 & 1164 & 2094 & 1164 & 1159 & 980 \\
\cline { 2 - 7 } & 2 & 890 & 1033 & 890 & 889 & 633 \\
\cline { 2 - 7 } & 3 & 597 & 561 & 561 & 597 & 472 \\
\hline
\end{tabular}

Table VIII: Average savings from adopting optimised strategies in the 36 trials and networks subject to different conditions.

\begin{tabular}{|l|l|l|l|l|}
\hline Condition & Scenario & $\begin{array}{l}\text { OTrad savings } \\
\text { compared } \\
\text { with: }\end{array}$ & $\begin{array}{l}\text { OSmart savings compared } \\
\text { with }\end{array}$ \\
\cline { 3 - 5 } & & Traditional & Smart & OTrad \\
\hline \multirow{3}{*}{ Normal } & 1 & $0.5 \%$ & $19 \%$ & $18 \%$ \\
\cline { 2 - 5 } & 2 & $0.1 \%$ & $27 \%$ & $34 \%$ \\
\cline { 2 - 5 } & 3 & $0.1 \%$ & $16 \%$ & $21 \%$ \\
\hline \multirow{2}{*}{$\begin{array}{l}\text { costs line } \\
\text { cond }\end{array}$} & 1 & $0.2 \%$ & $20 \%$ & $20 \%$ \\
\cline { 2 - 5 } & 2 & $0 \%$ & $27 \%$ & $37 \%$ \\
\hline \multirow{2}{*}{\begin{tabular}{c} 
costs DR \\
\cline { 2 - 5 }
\end{tabular}} & 2 & $0 \%$ & $16 \%$ & $18 \%$ \\
\cline { 2 - 5 } & 3 & $0.5 \%$ & $16 \%$ & $15 \%$ \\
\hline
\end{tabular}

scenario) and network configurations (i.e., radial or ring) and reinforcements and (iv) performing sequential Monte Carlo simulations (in average 400000 contingencies are simulated per network) based on the different profiles, configurations, reinforcements and DR levels. It is worth highlighting that these studies can be performed in parallel (for different networks and conditions), which can significantly reduce computational time if suitable computing resources are available.

The time required for the studies is clearly acceptable for planning purposes. However, this may not be true in other cases where networks of different sizes and different number of interventions and decision nodes may be considered. Accordingly, the sensitivity studies were repeated for the 36 networks available and assuming different number of interventions and decision nodes.

The size of the networks considered (ranging from 10 to 133 nodes) mainly impacts the time required to perform offline studies as shown in Figure 9. In this work, the time required for the off-line studies associated with most networks was less than 10 minutes, while longer computational time (roughly an hour) was required for three of the largest networks. The specific network configuration can of course also affect the load flow convergence, which can be seen in terms of "spikes" in Figure 9. This effect challenges the validity of particular trend lines to estimate the effects that larger networks may have on the time complexity of the algorithm. Regardless, taking a pessimistic point of view, it may be reasonable to assume exponential growth. Accordingly, based on an exponential curve fitted to the data presented in Figure 9, one hour and 24h of off-line studies are 


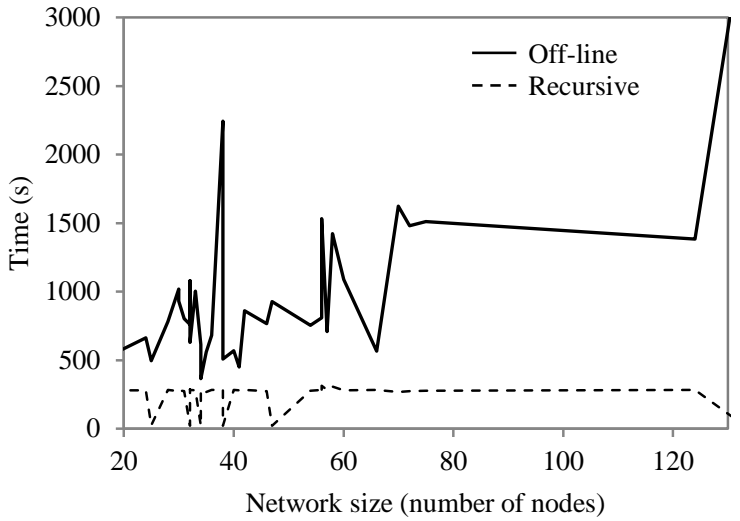

Figure 9: Time required for the off-line calculations and recursive function based on the size of the networks.

expected for the analysis of networks with roughly 150 nodes and 416 nodes, respectively. In general, it is important to emphasize that off-line computational time can vary significantly depending on the specific software used and dedicated power system simulation tools are likely to result in reduced computational time with respect to the use of Matlab (Matpower) as done here.

The results in Figure 9 also highlight that, due to the particular use of off-line studies, in this study, the impact of the size of the network on the time required by the recursive function is not significant. Nevertheless, the analysis of larger networks may require the consideration of an increased number of interventions. This can affect the computational complexity of the recursive function as explored below.

The results presented in Figure 10 show the time complexity of the recursive algorithm as a function of the number of decision nodes under consideration and interventions. The number of decision nodes indicate the periods (years in this example) within the planning horizon (45 years) when interventions can be made (i.e., $\mathrm{C}_{2} \mathrm{C}$, line reinforcements and substation upgrades in this exercise). For example, interventions can be made from year 1 to year 20 in the 20 decision nodes case. It is worth noting that, even though decision nodes can be placed in any other years (e.g., every two years from year 1 to year 39), in this work decision nodes have been placed annually at the beginning to the planning horizon because (i) interventions are seldom required in the long-term (i.e., after 20 years) due to the demand growth scenarios under consideration, (ii) intervention in the longterm have a low value due to discounting and (iii) the largest search space (and most computationally demanding analysis) can typically be found by placing the decision nodes at the beginning of the planning horizon.

The consideration of additional interventions is expected to increase the computational complexity of the algorithm, particularly if the interventions are interdependent (e.g., line reinforcements and the $\mathrm{C}_{2} \mathrm{C}$ method). In order to illustrate this effect, consider the flexibility that the $\mathrm{C}_{2} \mathrm{C}$ method provides to the distribution planner to defer line reinforcements. Similarly, line reinforcements provide headroom to defer implementing the $\mathrm{C}_{2} \mathrm{C}$ method. This effect increases the size of the feasible search space that the recursive function must explore
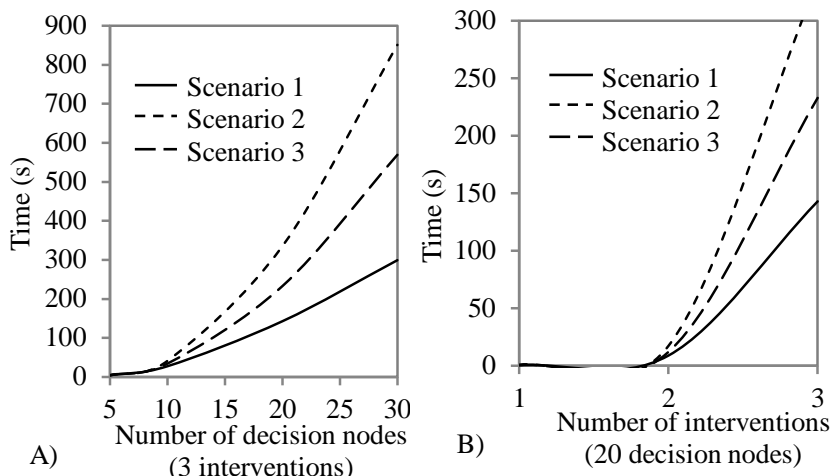

Figure 10: Time required to find optimal (e.g., OTrad and OSmat) investments based on a different number of $A$ ) decision nodes and $B$ ) interdenendent interventions.

compared with other cases with independent interventions (e.g., line and substation upgrades). Accordingly, with the aim of taking a pessimistic approach, the impact of the number of interventions on computational complexity is assessed using interdependent interventions. More specifically, one $\left(\mathrm{C}_{2} \mathrm{C}\right)$, two $\left(\mathrm{C}_{2} \mathrm{C}\right.$ and line reinforcement $)$ and three $\left(\mathrm{C}_{2} \mathrm{C}\right.$ and line and substation upgrades) interventions are considered.

It can be seen (from Figure 10) that the time required by the function to find a solution is heavily influenced by the characteristics of the scenarios. Fast demand growth scenarios (i.e., Scenario 1) require the deployment of solutions in the short-term, which in turn allow the algorithm to neglect significant sections of the search space and reduce computational burden. Conversely, planning the network in scenarios with little or no short-term demand growth (i.e., Scenario 2) requires the consideration of larger sections of the search space. Accordingly, as mentioned before, the proposed approach is more attractive for the planning of networks that may require upgrades in the short-term. It is worth noting that these impacts of the scenarios on the performance of the engine highlight the benefits from smartness to reduce the search space based on the conditions of the system. Without this intelligence, the performance of the exhaustive search would be independent of the scenario, as it would always explore all potential combination of conditions (potentially becoming computationally infeasible).

Based on the pessimistic data of Scenario 2, the relevant time complexity can be approximated with a quadratic function. This can be attributed to the ability of the algorithm to reduce the search space; otherwise, the growth would be exponential. Based on the quadratic growth, the proposed algorithm is expected to find optimal solutions within an hour for cases with up to 58 decision nodes or 6 interventions. The algorithm would also be expected to find solutions for up to 270 decision nodes and 50 interventions in roughly 24 hours. Taking a more pessimistic approach and assuming time complexity grows exponentially, the function is expected to find optimal solutions for studies with up to 35 decision nodes and 3 interventions within an hour, and up to 5 interventions or 50 decisions nodes in about 24 hours.

The results provide strong evidence that the time complexity of the algorithm is acceptable for planning 
purposes. In addition, the computational burden of the proposed function could further be reduced based on techniques (e.g., as those used with branch-and-bound algorithms [15][46]) to select the most relevant areas of the search space to explore first, and terminate instances before reaching the end of the planning horizon. Another modification to the algorithm that may reduce computational burden (requiring changing the algorithm so as to focus on the whole problem rather than on reduced sub-problems addressed by instances) is the use of iterations instead of recursions [38][39]. The abovementioned potential improvements to the proposed algorithm are left for future work.

\subsection{Additional applications}

The studies presented so far highlight the robustness of the proposed recursive function to be applied under a wide range of conditions that are in line with current planning practices (e.g., considering independent scenarios and risk neutrality). However, the introduction of publically available optimisation engines such as the proposed recursive function may allow DNOs to perform more detailed and realistic (but complex) planning studies. More specifically, the proposed methodology allows the introduction of (i) bespoke models and constraints (as most optimisation software and engines do) and (ii) complex nonlinear, nonconvex and/or integer problems (e.g., the power flow constraints and DR intervention models), which can be challenging to address by commercial software.

Based on the above and as an illustrative example of how planning practices could evolve in light of the availability of flexible optimisation engines, the proposed recursive function is extended to explicitly address uncertainty and risks.

In this work, uncertainty is modelled under the rationale that demand growth can be reasonably predicted in the short term, whereas it can change substantially in the long term. This is consistent with realistic conditions faced by DNOs as emerging new solutions that can greatly impact the distribution level in the long term (e.g., deployment of energy efficiency, electric vehicles, renewables and so forth), would take several years to materialise and would, thus, have a negligible impact in the short term. Accordingly, DNOs may be interested in investment strategies that are robust enough to meet demand growth in the short term, and flexible enough to be adjusted (at low costs) to the scenario that materialises in the long term. These types of flexible investments can be produced with the proposed recursive function if extended with scenario trees [47][48].

A scenario tree uses a limited number of scenarios (one in this case) to model short term uncertainty, whereas long term uncertainty is modelled by dividing the scenarios into several branches (three in this case). Such a tree (see Figure 11) is included in the proposed recursive function by modelling demand $\left(\operatorname{Dem}_{y}\right)$ with (10) during a single (low uncertainty) scenario that lasts $T$ years (5 years in this example), while using (11) to create branches connecting to three different (high uncertainty) demand scenarios right after year $T$ (i.e., $D_{1, y}, D_{2, y}$ and $D_{3, y}$ ), and using (12) to allow demand growth to follow the specific scenarios afterwards.

$$
\begin{gathered}
\operatorname{Dem}_{\text {Scen }, y}=D_{\text {Scen }, y} \quad \forall y \leq T, \text { Scen }=1 \\
\operatorname{Dem}_{\text {Scen }, y}=\left\{\begin{array}{l}
D_{1, y} \\
D_{2, y} \\
D_{3, y}
\end{array}\right\} \quad \forall y=T+1, \text { Scen }=1 \\
\operatorname{Dem}_{\text {Scen }, y}=D_{\text {Scen }, y} \quad \forall y>T+1, \text { Scen } \in[1,3]
\end{gathered}
$$

After the aforementioned modifications, the recursive function and its instances now create "child" instances in light of available scenarios (as well as available interventions) in every year and the "parent" instances map the scenario tree based on the feedback provided by each "child" instances ${ }^{2}$. Accordingly, considering the aforementioned scenario tree and the recursive function in optimisation mode, it is recommended to deploy a DR intervention in year one. Afterwards, it is recommended to reinforce the lines in year 6 (double reinforcement) and upgrade the substation in year 9 in Scenario 1, and reinforce the lines (single reinforcement) in year 9 in Scenario 2 (see Figure 11).

This strategy uses DR to manage demand growth in the short term, while providing flexibility for the DNO to avoid further investments (Scenario 3), perform low cost interventions (Scenario 2) or costly interventions (Scenario1) in response to the unfolding of uncertainty. The investment strategy results in NPCs of $799 £ \times 10^{3}, 528 £ \times 10^{3}$ and 466 $£ \times 10^{3}$ in Scenario 1, Scenario 2 and Scenario 3, respectively. That is, the average expected NPC of the investment strategy based on this particular tree is $598 £ \times 10^{3}$ (also assuming that all scenarios have the same probability of occurrence). These results can be replicated using the case study file provided in [22].

The abovementioned strategy is the most attractive under uncertainty in terms of the average (risk neutral) NPC. However, in practice, the DNO may be risk averse and prefer strategies that minimise costs in the worst case scenario (i.e., $799 £ \times 10^{3}$ in Scenario 1 in this example). In such case, risk management tools such as, for example, the opportunity cost

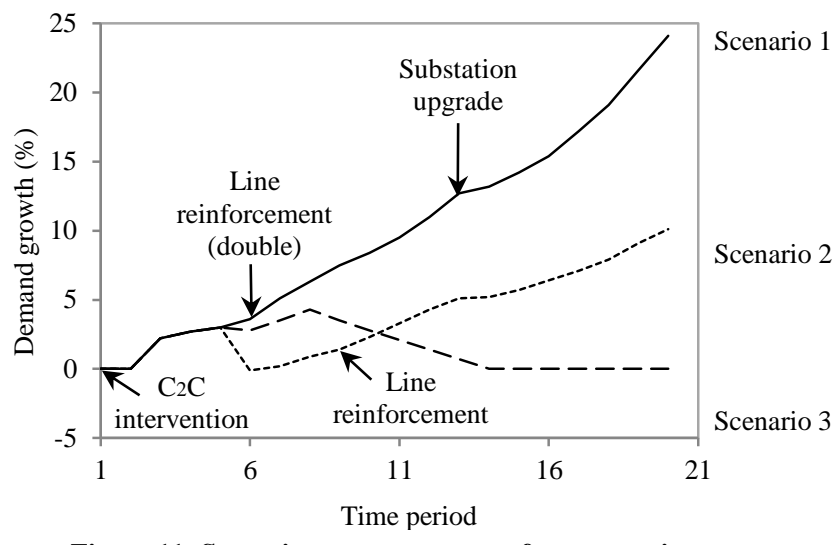

Figure 11: Smart investment strategy for a scenario tree.

\footnotetext{
${ }^{2}$ In other words, the recursive function can adapts to any type of scenario tree, regardless of its shape.
} 
constraints represented by (13) and (14) (see [47][49] for more details) can be implemented as part of the internal processes of the recursive function in optimisation mode. Accordingly, a limit (Limit) can be imposed on the opportunity cost $\left(C \_O p_{\text {Scenario } 1}\right)$, namely the maximum NPC increase in the worst case scenario (Scenario 1 in this example) associated with optimising the investment strategy in light of the full tree $\left(N P C_{\text {Scenario 1 } 1}^{\text {Tree }}\right)$ instead of the specific scenario $\left(N P C_{\text {Scenario } 1}^{\text {Smart }}\right)$. The introduction of such a constraint results in the formulation of the investment strategy presented in Figure 12.

$$
\begin{gathered}
C_{-} O p_{\text {Scenario } 1}=N P C_{\text {Scenario } 1}^{\text {Tree }}-N P C_{\text {Scenario } 1}^{\text {Smart }} \\
C_{-} O p_{\text {Scenario } 1} \leq \text { Limit }
\end{gathered}
$$

In this case, the NPC in the worst case scenario (Scenario 1) has been reduced to $795 £ \times 10^{3}$ (note that the minimum NPC in this scenario is $794 £ \times 10^{3}$ ), at the expense of increasing the expected NPC in Scenario 2 (now $545 £ \times 10^{3}$ ) and Scenario 3 (now $487 £ \times 10^{3}$ ), as well as the average NPC (now 609 $£ \times 10^{3}$ ). These results can also be replicated using the case study file provided in [22].

It is worth emphasizing that the recursive function effectively operates as flexible optimisation software for distribution network planning. Accordingly, a wide range of interventions, constraints, scenarios and so forth can be easily modelled by including the relevant models in the provided open source recursive function model [22].

\section{CONCLUSION}

This work has presented a distribution network planning methodology based on recursive functions and provided as an open-source Matlab tool that can emulate traditional businessas-usual distribution planning practices and even optimise them, also considering the presence of smart solutions such as DR. The tool is based on exhaustive algorithms (typically overlooked due to their high computational burden) that have been extended through recursion theory to systematically reduce the search space and, thus, computational costs. This makes the methodology simple, easy to understand and highly powerful at the same time, facilitating its practical use by DNOs and therefore reaching outside the more traditional academic realm.

A wide range of potential applications of the proposed recursive function have been illustrated based on real UK networks and the actual implementations of post-contingency DR for network support in the $\mathrm{C}_{2} \mathrm{C}$ project. Different scenarios and cases subject to current planning practices (presented as sensitivity studies) and new problems and proposed planning practices (i.e., considering scenario trees and risk management constraints) have been presented to illustrate the robustness and flexibility of the models proposed.

The results show that the recursive function based on the business-as-usual is an improvement to traditional planning practices as it can systematically formulate and assess investment strategies. This process is currently done manually

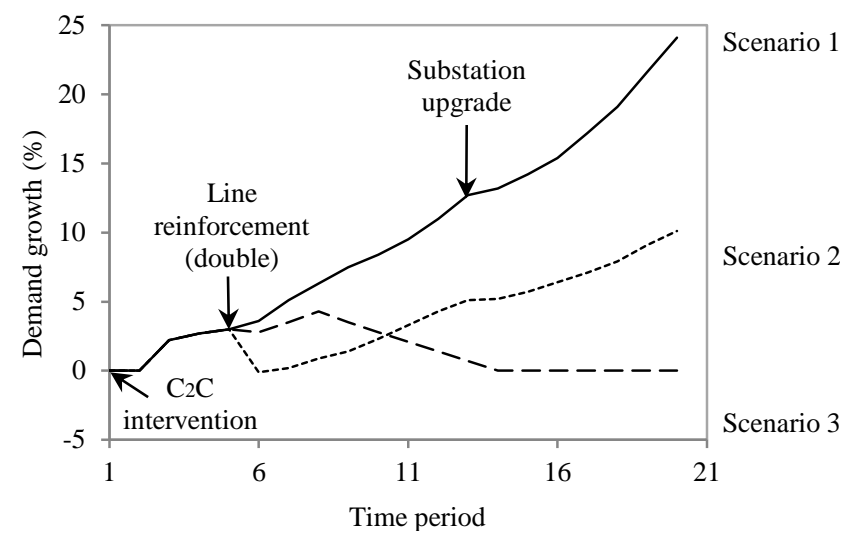

Figure 12: Smart investment strategy for a scenario tree considering a constraint for the maximum NPC in Scenario 1.

for a limited number of investment strategies. Furthermore, the recursive function can also improve the investment strategies by deploying an optimisation algorithm, which results in significant economic and social benefits particularly in light of smart active solutions (e.g., DR). The results also demonstrate that the proposed tool is robust enough to handle different types of networks under diverse conditions, as well as flexible enough to allow the consideration of additional constraints and considerations (e.g., scenario trees and risk management constraints).

Work in progress aims at exploring detailed models for emerging smart interventions and new distribution planning practices, particularly to address uncertainty in more detail.

\section{ACKNOWLEDGEMENTS}

The authors are grateful to Victoria Turnham, Paul Turner and Dr. Rita Shaw from ENWL for the continuous support and feedback provided during the $\mathrm{C}_{2} \mathrm{C}$ project. The authors would also like to thank Ms Angeliki Syrri for her inputs from reliability studies.

\section{REFERENCES}

[1] J. Varela, L. J. Puglisi, T. Wiedemann, U. Ysberg, D. Stein, Z Pokorna, C. Arnoult, R. Garaud-Verdier, L. Consiglio. Show Me!: Large-Scale Smart Grid Demonstrations for European Distribution Networks. IEEE Power and Energy Magazine, 2015;13(1):84 -91.

[2] A. Ipakchi, F. Albuyeh. Grid of the future. IEEE Power and Energy Magazine, 2009;7(2):52 -62.

[3] A. Navarro-Espinosa and P. Mancarella, Probabilistic modelling and assessment of the impact of electric heat pumps on low voltage electrical distribution networks, Applied Energy, 2014;127:249 -266.

[4] J. Schachter and P. Mancarella, Demand Response Contracts as Real Options: A Probabilistic Evaluation Framework under Short-Term and Long-Term Uncertainties, IEEE Transactions on Smart Grid, accepted for publication, February 2015.

[5] S. Altaher, J. Mutale, and P. Mancarella, Automated Demand Response from Home Energy Management System under Dynamic Pricing and Power and Comfort Constraints, IEEE Transactions on Smart Grid, 2015;6(4):1874 - 1883.

[6] E.A. Martinez-Cesena, N. Good, and P. Mancarella, Electrical Network Capacity Support from Demand Response: Techno-Economic Assessment of Potential Business Cases for Commercial and Residential End-Users, Energy Policy, 2015;82:222 - 232.

[7] J. R. Agüero. Tools for Success. IEEE Power and Energy Magazine, 2011;9(5):82-93. 
[8] R. F. Arritt, R. C. Dugan. Distribution System Analysis and the Future Smart Grid. IEEE Transactions on Industry Applications, 2011;47(6): $2343-50$.

[9] J. F. Franco, M. J. Rider, R. Romero. A mixed-integer quadraticallyconstrained programming model for the distribution system expansion planning. International Journal of Electrical Power \& Energy Systems, 2014;62: $265-72$.

[10] H. Ahmadi, J. R. Marti. Distribution System Optimization Based on a Linear Power-Flow Formulation. IEEE Transactions on Power Delivery, 2015;30(1):25 -33.

[11] A. Battegay, N. Hadj-Said, G. Roupioz, F. Lhote, E. Chambris, D. Boeda, L. Charge. Impacts of direct load control on reinforcement costs in distribution networks. Electric Power Systems Research,2015;120: $70-79$.

[12] F. Ding, K. A. Loparo. Hierarchical Decentralized Network Reconfiguration for Smart Distribution Systems-Part I: Problem Formulation and Algorithm Development. IEEE Transactions on Power Systems, 2015;30(2), $734-43$.

[13] M. S. Nazar, M. R. Haghifam. Multiobjective electric distribution system expansion planning using hybrid energy hub concept. Electric Power Systems Research, 2009;79(6):899 -911.

[14] S. Favuzza, G. Graditi, M. G. Ippolito, E. R. Sanseverino. Optimal Electrical Distribution Systems Reinforcement Planning Using Gas Micro Turbines by Dynamic Ant Colony Search Algorithm. IEEE Transactions on Power Systems, 2007;22(2):580 -7.

[15] ENWL. About C2C. 2015. Available from: $<$ http://www.enwl.co.uk/c2c >.

[16] E. A. Martinez Cesena and P. Mancarella, "Distribution network capacity increase via the use of demand response during emergency conditions: A cost benefit analysis framework for techno-economic appraisal," in CIRED 2014, 11 - 12 June, Italy, 2014.

[17] E. A. Martinez Cesena and P. Mancarella, "Economic assessment of distribution network reinforcement deferral through post-contingency demand response," in ISGT 2014, 12 -15 October, Turkey, 2014.

[18] G. Ücoluk, S. Kalkan, S, 2012. Introduction to programming concepts with case studies in python. Austria, Vienna:Springer.

[19] G. Dowek, G., 2009. Principles of Programming Languages. London:Springer.

[20] V. Maniezzo, T. Stützle, S. Voß, S, 2010. Matheuristics: Hybridizing metaheuristics and mathematical programming. Boston, MA:Springer.

[21] MathWorks, Matlab, 2015. Available from: <http://uk.mathworks.com/products/matlab/ >.

[22] Recursive function: Open source files. Available from: <https://www.dropbox.com/sh/6dwa1wrrssmye7t/AACrUPjAoNcGqm ov35oLcBaSa?dl=0>

[23] R. Allan, G. Strbac, P. Djapic, K. Jarrett, 2013. Developing the P2/6 Methodology. UMIST.

[24] Ofgem. Electricity distribution annual report for 2010-11. 2012.

[25] Ofgem. Strategy consultation for the RIIO-ED1 electricity distribution price control: Outputs, incentives and innovation. 2012.

[26] Ofgem. Template CBA RIIO ED1 v4. 2013.

[27] Ofgem. Strategy decision for the RIIO-ED1 electricity distribution price control: Business plans and proportionate treatment. 2013. Available from: <https://www.ofgem.gov.uk/ofgempublications/47069/riioed1decbusinessplans.pdf $>$.

[28] Ofgem. Network innovation. 2015. Available from: <https://www.ofgem.gov.uk/network-regulation-\%E2\%80\%93-riiomodel/network-innovation>.

[29] R. Bidwell, S. Darcy, N. Jenkins, D. Newberry, S. Sutcliffe, 2012. Report and recommendations prepared for the gas \& electricity markets authority. Available from: <http://www.enwl.co.uk/docs/c2c-keydocuments/low-carbon-networks-fund-2011-final-

report.pdf?sfvrsn=6>.

[30] P. Mancarella, C.K.Gan and G.Strbac, Optimal design of low voltage distribution networks for $\mathrm{CO} 2$ emission minimisation. Part I: Model formulation and circuit continuous optimisation, IET Generation Transmission and Distribution, 2011;5(1):38-46.

[31] P. Mancarella, C. K. Gan and G. Strbac, Optimal design of low voltage distribution networks for CO2 emission minimisation. Part II: Discrete optimisation of radial networks and comparison with alternative design strategies, IET Generation Transmission and Distribution, 2011;5(1):47 -56 .

[32] S. Blair and C. Booth, Analysis of the technical performance of C2C operation for HV Networks, 2014. Available from: <http://www.enwl.co.uk/docs/default-source/c2c-key-

documents/technical-performance-report.pdf?sfvrsn=4>.

[33] ENWL, Fault Restoration performance on HV rings as part of the capacity to customers project, ENWL, 2015. Available from: <http://www.enwl.co.uk/docs/default-source/c2c-key-documents/whitepaper---fault-performance-of-hv-rings.pdf?sfvrsn=10>.

[34] ENWL. High energy users. 2015. Available from: <http://www.enwl.co.uk/c2c/are-you-affected/high-energy-users>.

[35] V. Turnham and P. Turner, Capacity to customers second tier LCN fund project closedown report. 2015. Available from: <http://www.enwl.co.uk/docs/default-source/c2c-key-documents/c2cclosedown-report-aug-2015.pdf?sfvrsn=4>.

[36] G. E. Williamson and P. Turner, "Trial circuit selection methodology: capacity to customers (C2C) Project," ENWL. 2012. Available from: <http://www.enwl.co.uk/docs/default-source/c2c-key-documents/trialcircuit-selection-methodology1F73D00A0AD1.pdf?sfvrsn=10>.

[37] M. Brainch, K. Quigley and P. Turner, Capacity to customers customer survey: Post-acceptance survey report, ENWL, 2015 Available from: <http://www.enwl.co.uk/docs/default-source/c2c-keydocuments/customer-post-acceptance-surveyreport.pdf?sfvrsn $=42015>$.

[38] I. Skiliarova and V. Sklyrov, Recursive versus iterative algorithms for solving combinatorial search problems in hardware, in $21^{\text {st }}$ International conference on VLSID design, 4 -8 Jan. India, 2008.

[39] R. Cavallo and J. de Voy, Iterative and recursive algorithms for tree search and partition search of the lattice of structure models, International Journal of General Systems, 1992;20(3):275 - 301.

[40] SP Energy Networks, SP Energy Networks 2015-2023 Business plan: Annex Cost Benefit Analysis. 2014. Available from: <http://www.spenergynetworks.co.uk/userfiles/file/201403_SPEN_Cos tBenefitAnalysis_JW_Public.pdf $>$.

[41] E.-G. Talbi. Hybrid Metaheuristics. Berlin, Heidelberg:Springer:2013

[42] M.-C. Alvarez-Herault, N. N'Doye, C. Gandioli, N. Hadjsaid, P. Tixador. Meshed distribution network vs reinforcement to increase the distributed generation connection. Sustainable Energy, Grids and Networks, 2015;1:20-27.

[43] E. A. Martínez Ceseña and P. Mancarella, 2014. Sensitivity Analysis of the Expected Economic Value of the $\mathrm{C}_{2} \mathrm{C}$ method, The University of Manchester.

[44] PSERC. Matpower. 2014. Available from: <http://www.pserc.cornell.edu//matpower/>

[45] A. L. A. Syrri and P. Mancarella. Reliability evaluation of demand response to increase distribution network utilization. Proceedings of the 2014 International Conference on Probabilistic Methods Applied to Power System; 2014 July 7 -10; Durham, UK.

[46] P. Somol., P. Pudil and J. Kittler. Fast branch \& bound algorithms for optimal feature selection, IEEE Transactions on Pattern Analysis and Machine Intelligence, 2004;26(7):900-912.

[47] E. A. Martinez Cesena and P. Mancarella, Distribution network reinforcement planning considering demand response support, in PSCC 2014, 18 - 22 August, Poland, 2014.

[48] G. Infranger, Stochastic Programming: The state of the art, Springer, 2011.

[49] K-J. Miescke and F. Liese, Statistical Decision Theory, Springer, 2008. 